\title{
Singularity-Free Superstar as an Alternative to Black Hole and Gravastar
}

\author{
Ding-Yu Chung ${ }^{1}$, Volodymyr Krasnoholovets ${ }^{2}$ \\ ${ }^{1}$ P.O. Box 180661, Utica, USA \\ ${ }^{2}$ Institute of Physics, National Academy of Sciences, Kyiv, Ukraine \\ Email: dy_chung@yahoo.com,krasnoh@iop.kiev.ua
}

Received April 21, 2013; revised May 23, 2013; accepted June 25, 2013

Copyright (C) 2013 Ding-Yu Chung, Volodymyr Krasnoholovets. This is an open access article distributed under the Creative Commons Attribution License, which permits unrestricted use, distribution, and reproduction in any medium, provided the original work is properly cited.

\begin{abstract}
Singularity-free superstar is proposed as a model for the collapse of large stars and for GRBs, and as an alternative to black hole and gravastar. Similar to a superconductor, a superstar contains extreme force fields that have non-zero momentum and non-zero wavelength to prevent the inactivation of force field at absolute zero and singularity (infinite interacting energy) at infinite density, respectively, based on the uncertainty principle. Emerging only at an extremely low temperature above absolute zero or an extremely high density below infinite density, extreme force fields are shortrange, and located in between a particle and its ordinary force fields (electromagnetic, weak, strong, and gravitational forces) to prevent the inactivation of force fields at absolute zero and singularity (infinite interacting energy) at infinite density in ordinary force fields. Extreme force fields are manifested as the bonds among electrons in a superconductor and among atoms in a Bose-Einstein condensate. When the stellar core of a large star reaches the critical extreme density during the stellar collapse, the stellar core is transformed into the super matter core with extreme force fields and ordinary force fields without singularity. A pre-superstar contains the super matter core, the ordinary matter region, and the thin phase boundary between the super matter core and the ordinary matter region. The stellar collapse increases the super matter core by converting the in falling ordinary energy and matter from the ordinary matter region into the super matter, and decreases the ordinary matter region. Eventually, the stellar breakup occurs to detach the ordinary matter region and the phase boundary from the super matter core, resulting in GRB to account for the observed high amount of gamma rays and the observed complex light curves in GRBs. Unlike black holes and gravastars that lose information, singularity-free superstars that keep all information exist.
\end{abstract}

Keywords: Black Hole; Superstar; Gravastar; Extreme Force Field; Uncertainty Principle; Singularity; Space Structure; Collapsar; Gamma Ray Burst; Neutron Star; Pair Instability Supernova; Stellar Breakup

\section{Introduction}

Black hole has been a standard model for the collapse of a large star. Singularity in black hole remains contentious. Gravastar (gravitational vacuum star) [1] by P. O. Mazurand and E. Mottolais is a model for the collapse of a large star without singularity. In gravastar, quantum effects would change space-time around a collapsing star, initiating a radical phase transition like when liquid water becomes ice, for the in falling matter. For gravastar, the phase transition involves the transformation into a "gravitational vacuum" with an interior de Sitter condensate surrounded by a Bose-Einstein condensate (BEC) bubble, similar to the transformation of a cloud of atoms into one huge "super-atom", a BEC at an extremely low tempera- ture above absolute zero degree. The $\mathrm{BEC}$ is prevented from complete collapse by the interior de Sitter condensate exerting a balance pressure outwards on the condensate. A thin phase boundary (shell) for the phase transition is in between the interior region and the exterior region.

In this paper, singularity-free superstar is proposed as a model for the collapse of large stars and GRBs (gamma ray bursts). Superstar is an alternative to black hole and gravastar, and is similar to superconductor that involves extreme force fields that are derived from the space structure [2-4]. An extreme force field in a superstar is an alternative to a gravitational vacuum in a gravastar. Extreme force fields have non-zero momentum and nonzero wavelength to prevent the inactivation of force field 
at absolute zero and singularity (infinite interacting energy) at infinite density, respectively. Emerging only at an extremely low temperature above absolute zero or an extremely high density below infinite density, extreme force fields are short-range, and located in between a particle and its ordinary force fields (electromagnetic, weak, strong, and gravitational forces) to prevent the inactivation of force fields at absolute zero and singularity (infinite interacting energy) at infinite density in ordinary force fields. Extreme force fields are manifested as the bonds among electrons in a superconductor and among atoms in a Bose-Einstein condensate.

A superstar is formed by the transformation of the stellar core of a large star into the super matter core with extreme and ordinary force fields without singularity at an extremely high density during the stellar collapse. Superstar relates to space structure, the uncertainty principle, extreme force field, black hole, gravastar, neutron star, supernova, collapsar, GRB, and pair instability supernova.

\section{The Space Structure and Extreme Force Field}

The conventional explanation of the hidden extra space dimensions is the compactization of the extra space dimensions. For example, six space dimensions become hidden by the compactization, so space-time appears to be four dimensional. Bounias and Krasnoholovets [5] propose another explanation of the reduction of $>4 \mathrm{D}$ space-time into 4D space-time by slicing $>4 \mathrm{D}$ space-time into infinitely many 4D slices surrounding the 4D core particle. Such slicing of $>4 \mathrm{D}$ space-time is like slicing 3D object into 2D object in the way stated by Michel Bounias as follows: "You cannot put a pot into a sheet without changing the shape of the 2-D sheet into a 3-D dimensional packet. Only a 2-D slice of the pot could be a part of sheet".

One way to describe the slicing of space dimension is to have the space structure consisting of attachment space and detachment space. Attachment space is the space attaching to an object, such as 3D attachment space attaching to a pot described above. Detachment space is the space cutting attachment space into numerous attachment space slices, such as detachment space cutting 3D attachment space into numerous 2D attachment space slices. Such 2D attachment space slices with attached objects, therefore, are separated by 2D detachment space gaps without attached objects.

In this paper, the digital space structure consists of attachment space (denoted as 1) and detachment space (denoted as 0). Attachment space attaches to object permanently with zero speed. Detachment space detaches from the object at the speed of light. Attachment space relates to rest mass, while detachment space relates to kinetic energy. Different stages of our universe have different space structures.

The combination of attachment space (1) and detachment space (0) brings about three different space structures: miscible space, binary partition space, and binary lattice space as below:

$(1)_{n}$

attachment + detachment

space space

$\stackrel{\text { combination }}{\longrightarrow}(10)_{n}$ binary lattice space,

$(1+0)_{n}$ miscible space, or

$(1)_{n}(0)_{n}$ binary partition space

Binary lattice space, $\left(\begin{array}{ll}1 & 0\end{array}\right)_{\mathrm{n}}$, consists of repetitive units of alternative attachment space and detachment space. Thus, binary lattice space consists of multiple quantized units of attachment space separated from one another by detachment space to account for ordinary force fields (electromagnetic, strong, weak, and gravitational force fields). In miscible space, attachment space is miscible to detachment space, and there is no separation of attachment space and detachment space to account for special relativity. Binary partition space, $(1)_{n}(0)_{n}$, consists of separated continuous phases of attachment space and detachment space to account for quantum mechanics and extreme force field.

As described in Reference [2], at the beginning of the current universe, the $10 \mathrm{~d}$ particle universe was sliced into six particles: 9d, 8d, 7d, 6d, 5d, and 4d equally by mass. Detachment space (0) involves in the slicing of dimensions. Attachment space is denoted as 1. For example, the slicing of $10 \mathrm{~d}$ particles into $4 \mathrm{~d}$ particles is as follows:

$$
\left(1_{4+6}\right)_{i}
$$

10d attachment space

$$
\begin{aligned}
& \left(1_{4}\right)_{i} \quad \sum_{1}^{6}\left(\left(0_{4}\right)\left(1_{4}\right)\right)_{j, 6} \\
& \stackrel{\text { slicing }}{\longrightarrow} \begin{array}{l}
4 \mathrm{~d} \text { core } \\
\text { attachment } \\
\text { space }
\end{array}
\end{aligned}
$$

The two products of the slicing are the 4d-core attachment space for core particle and 6 types of $4 \mathrm{~d}$ quantized units for ordinary force fields. The $4 \mathrm{~d}$ core attachment space surrounded by 6 types of many (j) 4d-quantized units corresponds to the core particle surrounded by 6 types of many small $4 \mathrm{~d}$ particles. The force fields are the force fields with binary lattice space.

The uncertainty principle for quantum mechanics is expressed as follows: 


$$
\sigma_{x} \sigma_{p} \geq \frac{\hbar}{2}
$$

The position, $x$, and momentum, $p$, of a particle cannot be simultaneously measured with arbitrarily high precision. The uncertainty principle requires every physical system to have a zero-point energy (minimum momentum) greater than zero, and to have a maximum energy equal or less than the energy at the minimum wavelength as the Planck length. The uncertainty principle has nonzero momentum and non-zero wavelength. In terms of the space structure, detachment space relating to kinetic energy as momentum is $\sigma_{p}$, and attachment space relating to space (wavelength) for a particle is $\sigma_{x}$. Neither detachment space nor attachment space is zero in the uncertainty principle.

Quantum mechanics for a particle follows the uncertainty principle. It is proposed that at the extreme conditions of absolute zero and infinite density, the binary lattice space for ordinary force fields (electromagnetic, strong, weak, and gravitational force fields) follows the certainty principle $\left(\sigma_{x} \sigma_{p}=1 / \infty \approx 0\right)$ instead of the uncertainty principle. At absolute zero with infinitesimal movement, all detachment space (momentum) in binary lattice space virtually ceases to exist, so the binary space as the force field collapses into infinite attachment space (wavelength) with infinitesimal momentum, resulting in the inactivation of force field. At infinite density to produce infinite interacting energy (infinite momentum) from the interaction among particles, all attachment space (rest mass) in the binary lattice space virtually ceases to exist, so the binary lattice space as the force field collapses into infinite detachment space (momentum) with infinitesimal wavelength, resulting in singularity as infinite interacting energy.

To prevent the inactivation of force fields at absolute zero and singularity (infinite interacting energy) at infinite density in ordinary force fields requires the presence of the special force fields that follow the uncertainty principle. The special force fields are "extreme force fields" that are in binary partition space. Binary partition space has one continuous detachment space and one continuous attachment space. The binary partition space for extreme force fields follows the uncertainty principle. Neither detachment space nor attachment space is zero in the binary partition space. To follow the uncertainty principle, extreme force fields have non-zero momentum and non-zero wavelength to prevent the inactivation of force field at absolute zero and singularity (infinite interacting energy) at infinite density, respectively.

At the critical temperature above absolute zero and the critical extreme density below infinite density, extreme force fields emerge in between particles and their ordinary force fields (electromagnetic, strong, weak, and gra- vitational force fields) to prevent the inactivation of force fields at absolute zero and singularity (infinite interacting energy) at infinite density in ordinary force fields as follows:

$$
\begin{aligned}
& \left(1_{4}\right)_{m} \quad \sum_{k=1}^{k}\left(\left(0_{4}\right)\left(1_{4}\right)\right)_{n, k} \\
& \text { core ordinary forces } \\
& \text { particles in binary lattice space } \\
& \text { extremely low temperature or high density } \\
& \left(1_{4}\right)_{m}\left\{\sum_{k=1}^{k}\left(0_{4}\right)_{n, k}\left(1_{4}\right)_{n, k}\right\}\left\{\sum_{k=1}^{k}\left(0_{4}\right)\left(1_{4}\right)_{n, k}\right\} \\
& \text { core extreme forces ordinary forces } \\
& \text { particles in binary partition in binary lattice } \\
& \text { space space }
\end{aligned}
$$

The whole system of core particles, extreme force fields, and ordinary force fields never reaches absolute zero and infinite density. Extreme force fields do not change the normal properties of ordinary force fields in the same system.

All extreme force fields are identical and short-range, and are the dominant force fields over ordinary force fields in the interior of core particles, such as superconductor and Bose-Einstein condensate. The Meissner effect is explained by the outward pressure of extreme force fields to eject applied magnetic fields from the interior of the superconductor as it transitions into the extreme force fields at nearly absolute zero temperature. In the BCS theory of superconductivity [6], the superconducting current is explained as a super fluid of Cooper pairs, pairs of electrons interacting through the exchange of phonons. In the explanation by extreme force, the Cooper pairs correspond to pairs of electrons interacting through the exchanges of extreme force bosons. The superfluid property of a Bose-Einstein condensate can also be explained by the atoms interacting through the exchanges of extreme force bosons. More details of superconductivity by extreme force field are described in Reference [3].

\section{Neutron Star and Supernova}

The formation of neutron star involves the core collapse of a large star. When a star with the initial mass of about 8 to 25 solar masses depletes its nuclear fuel, it has no outward radiation pressure to support its bulk. The core of the star collapses into a neutron star by fusing electrons and protons into neutrons, sending out huge numbers of neutrons. The neutrino shock wave from these neutrinos causes a violent expulsion of the surrounding material, resulting in supernova [7]. The collapse in terms of the compression from a large size progenitor to 
a very small neutron star leads to a fast-rotating neutron star with a high angular momentum and a strong magnetic field.

\section{Collapsar, GRB, and Pair Instability Supernovae}

Gamma-ray bursts (GRBs) are the flashes of focused gamma rays associated with extremely energetic explosions that have been observed in distant galaxies. The energy of a GRB is approximately equal to turning a star like the Sun into pure energy. GRB can be explained typically by collapsar (collapsed star) [8] that refers to a specific model for the gravitational collapse of a fastrotating star, resulting in a stellar mass black hole.

In the collapsar model, when a star with initial mass about 25 to 90 solar masses collapses into a fast-rotating black hole, the black hole immediately begins to pull in more stellar material, and very quickly a rotating disk of material as black hole accretion disk (BHAD) forms. The inner portion of the disk spins around the superstar at near light speed. With rotating conducting fluids, the BHAD creates a strong magnetic field. Because the inner portion of the BHAD is rotating more quickly than the outer portion, the magnetic field lines twist violently. This causes a jet of material to blast outward at almost the speed of light perpendicularly to the BHAD. The jet contains matter and antimatter in the form of electrons, positrons, and protons. The gamma rays are produced by the "internal shocks" as the collisions of the shells of matter and energy pushed by the jet.

One of the problems in the collapsar model of GRB is to explain how some gamma-ray bursts may convert as much as half or more of the explosion energy into gamma-rays [9]. Another problem in the collapsar model of GRB is to explain the complexity of the light curves of GRBs [10]. The duration of observable emission can vary from milliseconds to tens of minutes. The numbers, the shapes, and the intensities of the peaks in the light curves vary. No two light curves are identical.

A hypernova [11] is a type of supernova with energy much higher than standard supernovae. One of the models for hypernova is pair instability supernova. Pair-instability supernova occurs in stars with an initial mass range from around 130 to 250 solar masses. The stellar core is occupied by gamma rays whose outward pressure keeps the star from collapse by the inward gravity. Electron-positron pairs can be created from gamma rays, resulting in the reduction of outward pressure by the decrease of gamma rays. This outward pressure drop leads to a partial collapse, resulting in an accelerated thermonuclear burning in a runaway thermonuclear explosion which blows the star completely apart without leaving a star remnant behind. The result is a hypernova.
For the star with an initial mass of 100 to 130 solar masses as in Eta Carinae [12], the partial collapse is not large enough to cause a runaway thermonuclear explosion. The thermonuclear explosion to only leads to the ejection of a part of outer layer. The repetition of the partial collapse finally depletes enough mass, resulting in a normal supernova. For a star with an initial mass higher than about 250 solar masses, the energies from the thermonuclear reactions are absorbed in photodisintegration. The stellar collapse continues without explosion.

\section{Superstar}

When a star with initial mass of about 25 to 90 solar masses collapses, the huge amount of collapsing materials allows the neutrino shock wave to have a weak or no supernova. The stellar collapse by inward gravity continues. The fast-rotating star resulted from the stellar collapse creates a strong magnetic field.

The stellar core is a small size gamma ray core. The outward pressure from this small size gamma ray core is too weak to stop the stellar collapse. The stellar collapse continues. When the stellar core reaches the critical extreme density by the stellar collapse, the stellar core is transformed into the super matter core with extreme force fields that prevent singularity in ordinary force fields by infinite density.

For a pre-superstar, the ordinary matter region is outside of the super matter core. The phase boundary is in between the super matter core and the ordinary matter region. The phase boundary is for the phase transition from ordinary matter to super matter. As in the Meissner effect to repel applied magnetic field in short-range, the super matter core exerts an outward pressure to repel the phase boundary in short-range with the strength proportional to the total mass of the super matter.

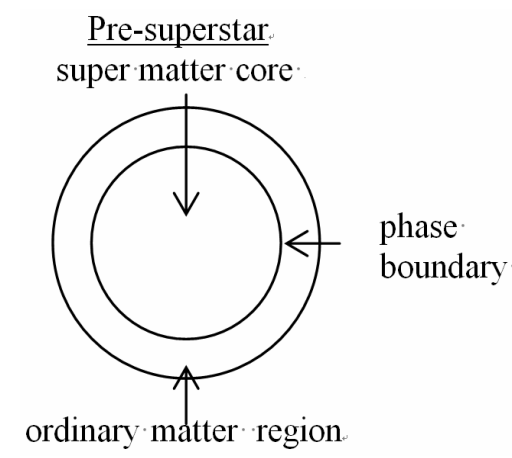

During the stellar collapse, the in falling energy that reaches the phase boundary is stored first as gamma rays in the phase boundary that is repelled by the super matter core. The in falling matter particles that reach the phase boundary are also stored first in the phase boundary. The density in the phase boundary is less than the critical 
extreme density. The further stellar collapse that increases the density in the phase boundary to the critical extreme density converts the gamma rays and matter particles in the phase boundary into super matter particles that move to the super matter core. The whole conversion process then starts over again. As a result, the stellar collapse increases the super matter core, and decreases the ordinary matter region.

Eventually, the ordinary matter region becomes small, and the inward gravity of the ordinary matter region is too weak to allow the ordinary matter region and the phase boundary to attach to the super matter core that repels the phase boundary. The result is the stellar breakup to detach the phase boundary and the ordinary matter region from the super matter core. The stellar breakup starts from the phase boundary that is repelled by the super matter core. During the stellar breakup, the detached phase boundary and ordinary matter region that are broken into pieces by the fast-rotation become the superstar accretion disk (SAD) as the black hole accretion disk (BHAD) in the collapsar model of GRB. With the additional energy from the gamma rays in the phase boundary, the SAD contains much higher energy than the BHAD. As the BHAD, the SAD produces a jet of material in the forms of electrons, positrons, and protons to blast outward at almost the speed of light perpendicularly to the SAD. The higher energy SAD produces the higher energy jet than the jet from the BHAD. The higher energy jet from the SAD produces more gamma rays from the internal shocks than gamma rays produced from the jet from the BHAD.

The different parts of the ordinary matter region in a compact superstar break up nearly simultaneously, so the GRB duration is short [13], and there is only one light peak in the light curve. The different parts of the ordinary region in a large superstar do not break up at the same time, so the GRB duration is long, more than one light peak are in the light curve, and different light peaks are different in intensities and shapes. The short duration GRBs have the average about 0.3 seconds and the longduration GRBs have the average about 30 seconds [14].

The observed high conversion of the explosion energy into gamma rays in a superstar breakup comes from the SAD that contains higher energy than the BHAD. For the light curves of GRBs, the additional complication that is not in the collapsar model is from the complex stellar breakup in a pre-superstar. Therefore, the superstar model of GRB solves the two problems of the collapsar model of GRB for the high conversion of the explosion energy into gamma rays and the complex light curves in GRBs. After the stellar breakup, the remnant is a pure superstar with only the super matter core. A pure superstar with a high gravity hinders the emission of light.

For the stellar breakup of a non-rotating superstar, en- ergies are not focused by the magnetic field of a fastrotating superstar. The stellar breakup is similar to a supernova.

For a star with an initial mass of 100 to 130 solar masses, the stellar core is a medium size gamma ray core that has a strong outward pressure to stop the stellar collapse and to prevent the formation of the super matter core. The core collapse by pair instability leads to a thermonuclear explosion, but not a runway thermonuclear explosion. The thermonuclear explosion leads to the ejection of a part of the ordinary matter region. For a star with an initial mass of 130 to 250 solar masses, the stellar core is a large size gamma ray core that has a strong outward pressure to stop the stellar collapse and to prevent the formation of the super matter core. The core collapse by pair instability leads to a runway thermonuclear explosion, resulting in a hypernova (pair instability supernova) without any star remnant. For a star with an initial mass of higher than about 250 solar masses, photodis integration prevents thermonuclear explosion, resulting in continuing stellar collapse to convert the stellar core into the super matter core for a supermassive pre-superstar.

From outside, black holes, gravastars, and superstars look the same. From inside, they are different in terms of information. An extreme force field that prevents singularity in gravity is an alternative to a gravitational vacuum (with the equation of state $p=-\rho$ ) in a gravastar. In a gravastar, a gravitational vacuum is located in one specific region. In a superstar, extreme force fields are not in one special region. The phase boundary in superstar is an alternative to the phase boundary in gravastar for the phase transition with equation of state $p=+\rho$ between the interior region and the exterior region (with the equation of state $p=\rho=0$ ). In a gravastar, in falling matter that hits the phase boundary is converted into energy by proton decay, adding to the energy of the space-time vacuum within the phase boundary. Some information such as baryon number conservation is lost during the transition from the exterior region to the interior region. In a black hole, all information other than the total mass, charge, and angular momentum is lost. In a superstar, all ordinary force fields in the super matter core are recoverable under ordinary condition, so no ordinary information is lost in a superstar.

Black holes and gravastars lose the information about ordinary force fields, while superstars keep all information about ordinary force fields. Quantum mechanics is built on the principle that information cannot be lost. Violating this basic principle of quantum mechanics, black holes and gravastars do not exist. In compliance with this basic principle, superstars exist.

\section{Summary}

It is proposed that the digital space structure consists of 
attachment space (denoted as 1) for rest mass and detachment space (denoted as 0 ) for kinetic energy. Attachment space attaches to object permanently with zero speed, and detachment space detaches from the object at the speed of light. The combination of attachment space and detachment space brings about the three structures: binary lattice space, miscible space, and binary partition space. Binary lattice space, $\left(\begin{array}{ll}1 & 0\end{array}\right)_{\mathrm{n}}$ consists of repetitive units of alternative attachment space and detachment space to account for ordinary force fields. In miscible space, attachment space is miscible to detachment space without separation to account for special relativity. Binary partition space, $(1)_{\mathrm{n}}(0)_{\mathrm{n}}$, consists of separated continuous phases of attachment space and detachment space to account for quantum mechanics and extreme force fields. Through the detachment space, a higher dimensional particle in attachment space is sliced into infinitely surrounding a lower dimensional core attachment space, resulting in a particle surrounding by force field in the form of binary lattice space. Under the extreme conditions of extremely low temperature or extremely high density, extreme force fields in binary partition space emerge.

Singularity-free superstar is proposed as a model for the collapse of large stars and for GRBs, and as an alternative to black hole and gravastar. Similar to a superconductor, a superstar contains extreme force fields that have non-zero momentum and non-zero wavelength to prevent the inactivation of force field at absolute zero and singularity (infinite interacting energy) at infinite density, respectively, based on the uncertainty principle. Emerging only at an extremely low temperature above absolute zero or an extremely high density below infinite density, extreme force fields are short-range, and located in between a particle and its ordinary force fields (electromagnetic, weak, strong, and gravitational forces) to prevent the inactivation of force fields at absolute zero and singularity (infinite interacting energy) at infinite density in ordinary force fields. Extreme force fields are manifested as the bonds among electrons in a superconductor and among atoms in a Bose-Einstein condensate. When the stellar core of a large star reaches the critical extreme density during the stellar collapse, the stellar core is transformed into the super matter core with extreme force fields and ordinary force fields without singularity. A pre-superstar contains the super matter core, the ordinary matter region, and the thin phase boundary between the super matter core and the ordinary matter region. The stellar collapse increases the super matter core by converting the in falling ordinary energy and matter from the ordinary matter region into the super matter, and decreases the ordinary matter region. Eventually, the stellar breakup occurs to detach the ordinary matter region and the phase boundary from the super matter core, resulting in GRB to account for the observed high amount of gamma rays and the observed complex light curves in GRBs. Unlike black holes and gravastars that lose information, singularity-free superstars that keep all information exist.

\section{REFERENCES}

[1] P. O. Mazur and E. Mottola, Proceedings of the National Academy of Sciences of the United States of America, Vol. 111, 2004, pp. 9545-9550. doi:10.1073/pnas.0402717101

[2] D. Chung and V. Krasnoholovets, Scientific Inquiry, Vol. 8, 2007, pp. 165-182.

www.iigss.net/scientific_inquiry/2007-12/3-krasnoholove ts.pdf

[3] D. Chung and V. Krasnoholovets, Progress in Physics, Vol. 4, 2006, pp. 74-77.

http://www.ptep-online.com/index_files/2006/PP-06-14.P DF

[4] D. Chung and V. Krasnoholovets, Journal of Modern Physics, Vol. 4, 2013, pp. 27-31.

[5] M. Bounias and V. Krasnoholovets, The International Journal of Systems and Cybernetics, Vol. 32, 2003, pp. 945-975.

[6] J. Bardeen, L. N. Cooper and J. R. Schrieffer, Physical Review, Vol. 108, 1957, pp. 1175-1205. doi:10.1103/PhysRev.108.1175

[7] S. Woosley and H.-T. Janka, Nature Physics, Vol. 1, 2005, pp. 147-154. doi:10.1038/nphys 172

[8] A. I. MacFadyen and S. Woosley, Astrophysical Journal, Vol. 524, 1999, pp. 262-289. doi:10.1086/307790

[9] Y. Fan and T. Piran, Monthly Notices of the Royal Astronomical Society, Vol. 369, 2006, pp. 197-206. doi:10.1111/j.1365-2966.2006.10280.x

[10] J. I. Katz, "The Biggest Bangs," Oxford University Press, Oxford, 2002, p. 37.

[11] G. S. Fraley, Astrophysics and Space Science, Vol. 2, 1968, pp. 96-114. doi:10.1007/BF00651498

[12] A. Kashi and N. Soker, New Astronomy, Vol. 14, 2008, pp. 11-24. doi:10.1016/j.newast.2008.04.003

[13] E. Nakar, Physics Reports, Vol. 442, 2007, pp. 166-236. doi:10.1016/j.physrep.2007.02.005

[14] C. Kouveliotou, et al., Astrophysical Journal Letters, Vol. 413, 1993, pp. 101-104. doi:10.1086/186969 